\title{
A Microwave-Sensitive Solid Acid Catalyst Prepared from Sweet Potato via a Simple Method
}

\author{
Hai-Ying Chen and Zheng-Wei Cui * \\ Jiangsu Key Laboratory of Advanced Food Manufacturing Equipment and Technology, \\ School of Mechanical Engineering, Jiangnan University, Wuxi 214122, Jiangsu, China; fly88honey@126.com \\ * Correspondence: cuizhengwei@jiangnan.edu.cn; Tel.: +86-510-8591-2082
}

Academic Editor: Ivan V. Kozhevnikov

Received: 16 October 2016; Accepted: 12 December 2016; Published: 17 December 2016

\begin{abstract}
In this study, a microwave-sensitive solid acid catalyst was successfully synthesized from sweet potatoes via a simple process. The catalyst was proven to have superior microwave-sensitive and homogeneous properties. The physicochemical properties were characterized by Brunauer-Emmett-Teller (BET), X-ray diffraction (XRD), Fourier-transform infrared spectra (FT-IR), thermogravimetric (TGA), scanning electron microscope (SEM) and elemental analysis (EA). Results showed that the total acid density and specific surface area for the catalyst were $6.35 \mathrm{mmol} / \mathrm{g}$ and $78.35 \mathrm{~m}^{2} / \mathrm{g}$, respectively. The elemental sulfur content reached $7.449 \%$ after sulfonation and the catalytic activity could reach over $91 \%$ within 30 min with microwave power density of $1.0 \mathrm{~W} / \mathrm{mL}$. The catalytic reaction temperature should not exceed $200{ }^{\circ} \mathrm{C}$, as shown in TGA curve, and the moisture content in the oil raw material should be within $1 \%-2 \%$. The catalyst deactivated gradually to $64.38 \%$ after reutilization five times, but the catalytic activity could be simply regenerated by re-sulfonation, albeit slightly reduced $(87.56 \%)$. The shift of diffraction peaks in the XRD patterns and new absorption peaks at 619.98 and $1190.49 \mathrm{~cm}^{-1}$ of FT-IR spectra demonstrated that the $-\mathrm{SO}_{3} \mathrm{H}$ group was effectively attached to the catalyst. The SEM images displayed a loose and porous amorphous structure in the end catalyst.
\end{abstract}

Keywords: solid acid catalyst; sweet potato; microwave sensitive; simple method

\section{Introduction}

In recent years, carbon-based solid acid catalysts have attracted attention because they provide a large number of strong acid sites, which can resist catalyst deactivation by water produced during the reaction. Additionally, these catalysts are low in cost and easily prepared [1-5]. Chen and Fang [6] prepared the catalyst from the glucose-starch mixture and also found that the amylopectin ratio in starch had an important effect on the formation of the small polycyclic aromatic carbon rings which provided anchoring points for sulfonic groups $\left(-\mathrm{SO}_{3} \mathrm{H}\right)$. Toda et al. [7] successfully produced carbon catalysts from carbonized starch and cellulose, which can be used as a replacement for liquid sulphuric acid in esterification reactions. Zong et al. [8] prepared a "sugar catalyst" from D-glucose and investigated its catalytic properties and structure in detail. The results indicated that this catalyst was highly suitable for the production of biodiesel from waste oils with high acid values. However, based on the analysis of the catalysts mentioned above, it was not difficult to find that almost all catalyst preparations need dry $\mathrm{N}_{2}$ protection. At the same time, most of the above solid acid-catalyzed biodiesel production processes experienced slower heat and mass transfer. Accordingly, higher temperatures, pressures and alcohol-to-oil molar ratios, along with longer reaction times are usually required to obtain higher esters yields due to the mass transfer resistance inside solid acid catalysts [9]. How to accelerate the reaction and improve process efficiency is, therefore, a major challenge for the use of the carbon-based solid acid catalyst. 
Microwave radiation has generated more and more attention due to its "magic" effects in chemical reactions. The special electromagnetic field effects and heating properties of microwave radiation allow chemical reaction systems to be heated quickly, bringing about necessary changes in molecular energy levels. Therefore, microwave radiation makes chemical reactions faster and reduces their energy demands, increasing efficiency and making them greener and more environmentally sound [10]. Some studies into the microwave-assisted synthesis of biodiesel confirmed that microwave radiation could accelerate the reaction [11-13]. Yuan et al. [14] prepared a $\mathrm{H}_{2} \mathrm{SO}_{4} / \mathrm{C}$ catalyst by the impregnation method, and their experimental results showed that the catalyst had a strong microwave absorption capacity, forming "microwave hot spots" under microwave radiation which were judged to be the reason for the significant acceleration of the reaction. Therefore, one purpose of this study was to produce a carbon-based solid acid catalyst which could absorb microwave energy and be suitable for applying to microwave radiation.

The sweet potato is rich in starch, which consists of amylose and amylopectin. The ratio of these two substances (amylose/amylopectin, 1:5) was close to that of glucose-corn starch (amylopectin $72 \%$ ) as reported by Chen and Fang [6]. Based on their research results, we conjectured that sweet potato starch, once carbonized, would possess large specific surface areas and more intensive internal network structures, providing a richer active site for the sulfonic acid reaction. Additionally, sweet potato is very inexpensive and widely available. For these reasons, the sweet potato is chosen as the potential carbon-based solid acid catalyst material in this study.

In this work, a microwave-sensitive solid acid catalyst was prepared through a simple method using sweet potato as the raw material. The microwave-sensitive and homogeneous properties of catalyst were investigated using the oleic acid as the model. The catalytic activity of the catalyst was investigated in the oleic acid-methanol reaction with different microwave power densities and water contents. The catalytic activity of this catalyst was also compared with concentrated sulfuric acid $\left(\mathrm{H}_{2} \mathrm{SO}_{4}\right)$ under conventional and microwave heating. The physicochemical properties of the catalyst were characterized using the X-ray diffraction (XRD), Brunauer-Emmett-Teller (BET), Fourier transform infrared spectrometry (FT-IR), thermogravimetric (TGA), scanning electron microscope (SEM) and elemental analysis (EA).

\section{Results and Discussion}

\subsection{Microwave Sensitive and Homogeneous Properties}

The homogeneous property of microwave heating was characterized using $20 \mathrm{~g}$ of oleic acid with $1 \mathrm{~g}$ of catalyst as the test model. Figure 1A shows the different position in the cylindrical resonant cavity of the microwave reactor. The temperature variations of the test model in the different nine points (P1-P9) were recorded in Figure 1. It was not difficult to find that the temperature difference in different position of the microwave resonant cavity could be ignored, with the exception of P5. The temperature at $\mathrm{P} 5$ was $6-10^{\circ} \mathrm{C}$ higher than at the other points (Figure 1). These phenomena could be explained through the following two aspects. On the one hand, P5 was set in the center of the cylindrical resonant cavity where the microwave energy was focused, and the temperature here was then a little higher than at other points. On the other hand, the similar temperature variations in points 1-9 illustrated that the catalyst in the test model did not cause the microwave energy to be reflected or refracted. Thus, the catalyst displayed a favorable homogeneous property and was suitable for the esterification reaction in the microwave reactor at P5. 


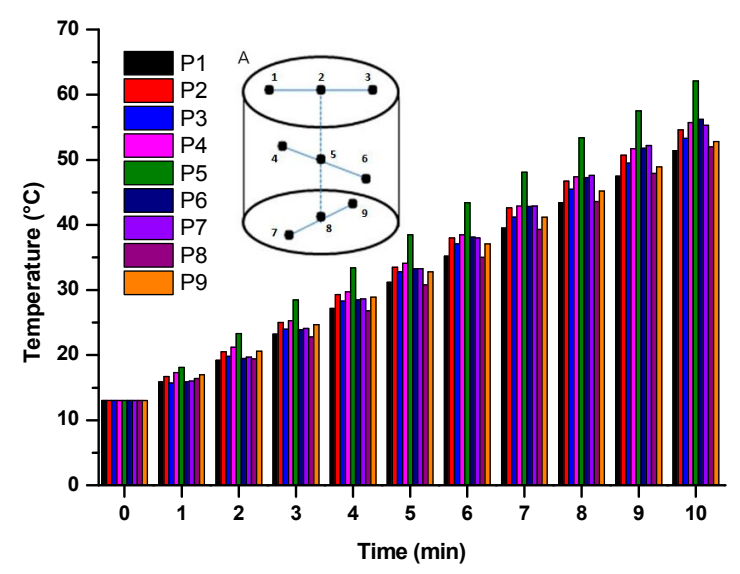

Figure 1. The temperature variation of each point (P1-P9) under microwave heating at $100 \mathrm{~W}$ for 10 min. A: the point location in cylindrical resonant cavity.

The microwave-sensitive property of the catalyst was characterized by the model of oleic acid with/without the catalyst under different microwave powers at P5. As shown in Figure 2, the temperature of $20 \mathrm{~g}$ of oleic acid under microwave heating at $100 \mathrm{~W}$ for $10 \mathrm{~min}$ was increased to $34.9^{\circ} \mathrm{C}$, while when $1 \mathrm{~g}$ of catalyst was added to $20 \mathrm{~g}$ of oleic acid, the temperature sharply increased to $56.6^{\circ} \mathrm{C}$. The increase trend was more significant at higher microwave powers. At 200 and $300 \mathrm{~W}$, the temperature increased from 47.0 to $90.5^{\circ} \mathrm{C}$ and from 73 to $110.9^{\circ} \mathrm{C}$, respectively. The heating rate was calculated and summarized in Table 1 . The heating rate of the test model with catalyst was 3.00, 6.58 , and $8.79{ }^{\circ} \mathrm{C} / \mathrm{min}$, respectively, with microwave heating at 100,200 and $300 \mathrm{~W}$. The higher heating rate demonstrated that the catalyst was more sensitive to microwave energy. This phenomenon could be ascribed to the catalyst composition, including the carbon framework, sulfonic acid group and carboxylic acid group, which was verified to absorb the microwave energy [15-17]. This excellent microwave-sensitive property would also be benefit for catalyzing esterification reaction.

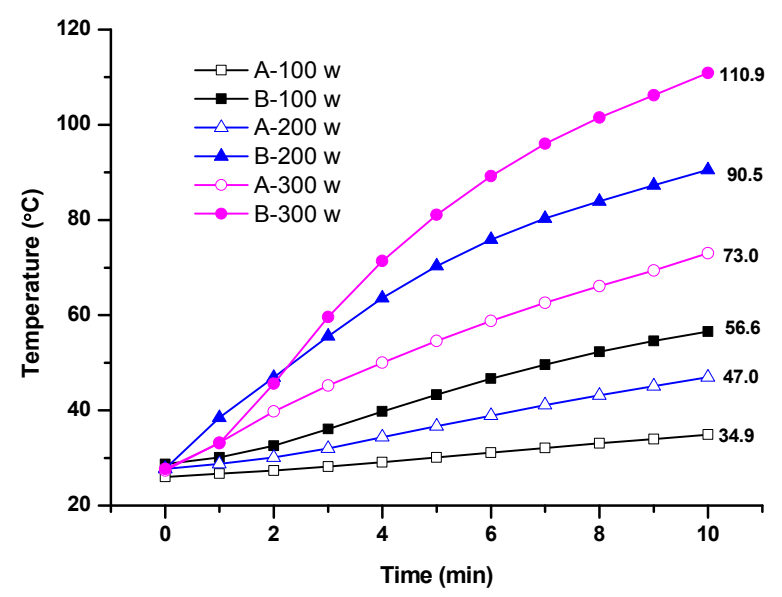

Figure 2. Curves of temperature for $20 \mathrm{~g}$ of oleic acid (A) and $20 \mathrm{~g}$ of oleic acid with $1 \mathrm{~g}$ of catalyst (B) at various microwave powers for different time.

Table 1. The heating rate of $20 \mathrm{~g}$ of oleic acid (A) and $20 \mathrm{~g}$ of oleic acid with $1 \mathrm{~g}$ of catalyst (B) at various microwave powers.

\begin{tabular}{ccccccc}
\hline \multirow{2}{*}{$\begin{array}{c}\text { Heating } \\
\text { Rate }\end{array}$} & \multicolumn{2}{c}{$\mathbf{1 0 0 \mathrm { W }}$} & \multicolumn{2}{c}{$\mathbf{2 0 0 \mathrm { W }}$} & \multicolumn{2}{c}{$300 \mathrm{~W}$} \\
\cline { 2 - 7 } & $\mathbf{A}\left({ }^{\circ} \mathrm{C} / \mathbf{m i n}\right)$ & $\mathbf{B}\left({ }^{\circ} \mathrm{C} / \mathbf{m i n}\right)$ & $\mathbf{A}\left({ }^{\circ} \mathbf{C} / \mathbf{m i n}\right)$ & $\mathbf{B}\left({ }^{\circ} \mathrm{C} / \mathbf{m i n}\right)$ & $\mathbf{A}\left({ }^{\circ} \mathbf{C} / \mathbf{m i n}\right)$ & $\mathbf{B}\left({ }^{\circ} \mathbf{C} / \mathbf{m i n}\right)$ \\
\hline $\mathrm{k}$ & 0.91 & 3.00 & 2.03 & 6.58 & 4.50 & 8.79 \\
\hline
\end{tabular}




\subsection{Catalytic Activity}

The catalyst prepared by the improved process in this study showed admirable catalytic activity (over 91\%) when compared to the results in previous related reports $[6,8,15,18-23]$. As shown in Table 2, the catalytic performance in catalyzing methanol/oleic acid in this study was slightly lower than the catalysts made from glucose-starch mixture [6], D-glucose [8], starch [18], corn straw [21] and seed shell [22], but higher than that from peanut shell [15], cellulose [18], activated carbon [19]. This phenomenon might be ascribed to the following two aspects. On one hand, the catalyst activity might be dependent on physicochemical properties (such as the content of starch, and the ratio of amylose and amylopectin) of the starting materials which determine the formation of polycyclic aromatic carbon rings and then the combination with the $-\mathrm{SO}_{3} \mathrm{H}$ group [18]. On the other hand, this difference might be caused by the different types of alcohol and fatty acids. The decreased activity with the long carbon-chain length of the fatty acids was due to the steric hindrance effect of the carbon chains [8] and the large specific surface area and mesoporous structures of the activated carbon [19]. At the same time, the dispersion of the catalysts in alcohol would also influence their catalyzing activity [24].

Table 2. The esterification activity of the catalyst in this study and other recent typical examples.

\begin{tabular}{cccc}
\hline Catalyst Material & Molar Ratio of Alcohol and Fatty Acid & Activity & Ref. \\
\hline Sweet potatoes & methanol/oleic acid 6:1 & $91 \%$ & This study \\
Glucose-starch mixture & methanol/oleic acid 10:1 & $96 \%$ & {$[6]$} \\
D-glucose & methanol/oleic acid 10:1 & $95 \%$ & {$[8]$} \\
Peanut shell & methanol/cottonseed oil 9:1 & $90.2 \%$ & {$[15]$} \\
Starch & methanol/oleic acid 10:1 & $95 \%$ & {$[18]$} \\
Cellulose & methanol/oleic acid 10:1 & $88 \%$ & {$[18]$} \\
Activated carbon & ethanol/acetic acid 10:1 & $78 \%$ & {$[19]$} \\
Phellinus igniarius & methanol/palmitate acid 10:1 & $91.5 \%$ & {$[20]$} \\
Corn straw & methanol/oleic acid 7:1 & $98 \%$ & {$[21]$} \\
Seed shells & methanol/oleic acid 1:1 & $95.7 \%$ & {$[22]$} \\
Rice husk & methanol/oleic acid 5:1 & $91 \%$ & {$[23]$} \\
\hline
\end{tabular}

\subsubsection{Effects of Microwave Power Density}

As shown in Figure 3A, the increase in microwave power density from 0.2 to $1 \mathrm{~W} / \mathrm{mL}$ caused a significant enhancement in ester yield from $72 \%$ to $91.6 \%$. However, when the microwave power density was further increased from 1 to $3 \mathrm{~W} / \mathrm{mL}$, the yield decreased from $91.6 \%$ to $84 \%$. The microwave output should not be too high, as it might cause a sharp rise in reaction temperature, resulting in more accumulated discontinuities and shortened molecular reorientation and vibration times. The microwave radiation was automatically turned on or off by temperature detection in these experiments to maintain the reaction temperature between 58 and $63{ }^{\circ} \mathrm{C}$ during the entire reaction process. In contrast, if microwave output was too low, most of the microwave power would be used to warm up the reaction mixture, and the temperature would be lower than required in most cases, resulting in a lower yield. The appropriate microwave power density and its dissipation control were very important to maximize ester yield and minimize reaction time and microwave energy consumption. 

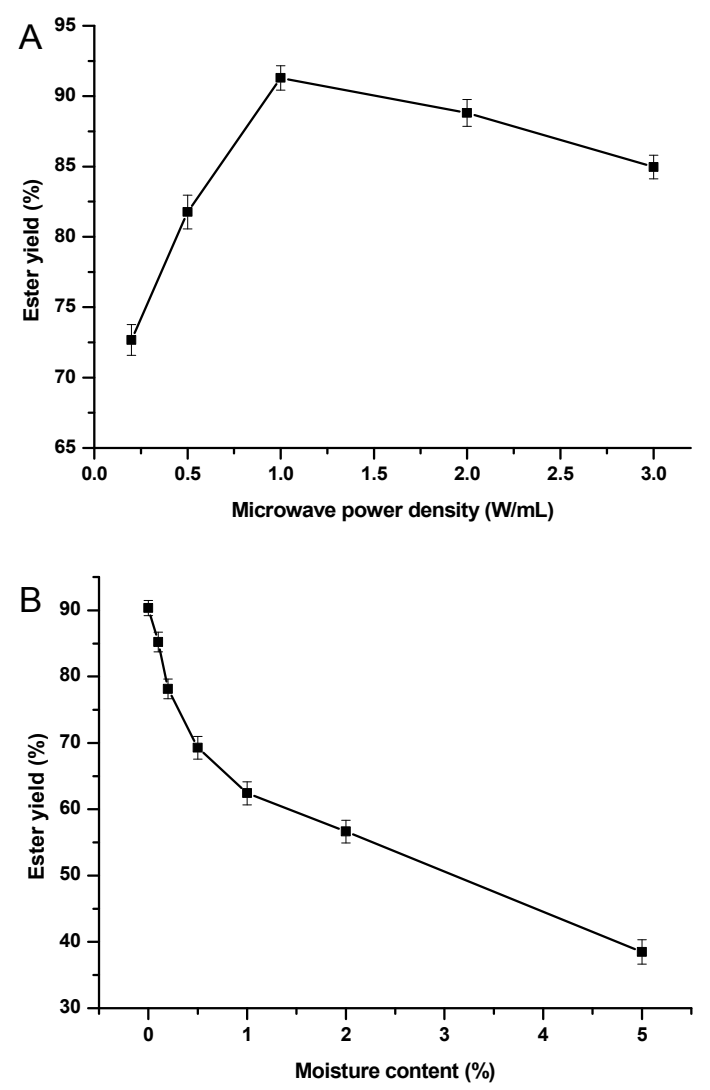

Figure 3. Effect of microwave power density (A) and moisture content (B) on ester yield. (A) reaction time, $15 \mathrm{~min}$; (B) microwave power density, $1 \mathrm{~W} / \mathrm{mL}$, reaction time, $15 \mathrm{~min}$.

\subsubsection{Effect of Moisture Content under Microwave Heating Environment}

It was well known that the water content in the reaction mixture was one of the most important factors affecting the esterification yield, due to saponification. The investigation was also carried out in the oleic acid-methanol model with a microwave power density of $1 \mathrm{~W} / \mathrm{mL}$ for $15 \mathrm{~min}$. The moisture content was set as 0, 0.1, 0.2, 0.5, 1, 2 and $5 \mathrm{wt} \%$ and the results are shown in Figure 3B. It was not difficult to find that a sharp decrease in ester yield occurred from $92 \%$ to $62 \%$ when the moisture content was less than $1 \mathrm{wt} \%$. Upon further increasing the moisture content from 1 to $5 \mathrm{wt} \%$, the ester yield decreased gradually from $62 \%$ to $40 \%$. This result demonstrated that the dehydration pretreatment of the raw material oils was a very important step if the cooked or low-quality oils were used as the raw materials for the production of biodiesel.

\subsubsection{Comparison of Catalyst Type and Reaction-Heating Method}

As can be seen in Figure 4A, the homogeneous concentrated sulfuric acid exhibited higher catalytic activity than the sulfonated carbon-based solid catalyst under conventional heating at $60{ }^{\circ} \mathrm{C}$. This result may be due to the fact that the concentrated sulfuric acid could be thoroughly mixed with the methanol and oleic acid, resulting in a faster reaction and a higher ester yield. With the depletion of concentrated sulfuric acid, the increase trend of ester yield slowed down after two hours of reaction. Regarding to the sulfonated carbon-based solid catalyst, the reaction rate was slow during the initial two hours because of the mass transfer resistance inside the catalyst. After that, the reaction rate increased quickly due to the decreased viscosity of mixture as the reaction proceeded. With the reaction time prolonged, the ester yield of the two catalysts became similar. This conclusion indicated that the current carbon-based solid catalyst could also produce high ester yields (compared to concentrated sulfuric acid), but would require a longer reaction time under conventional heating. 

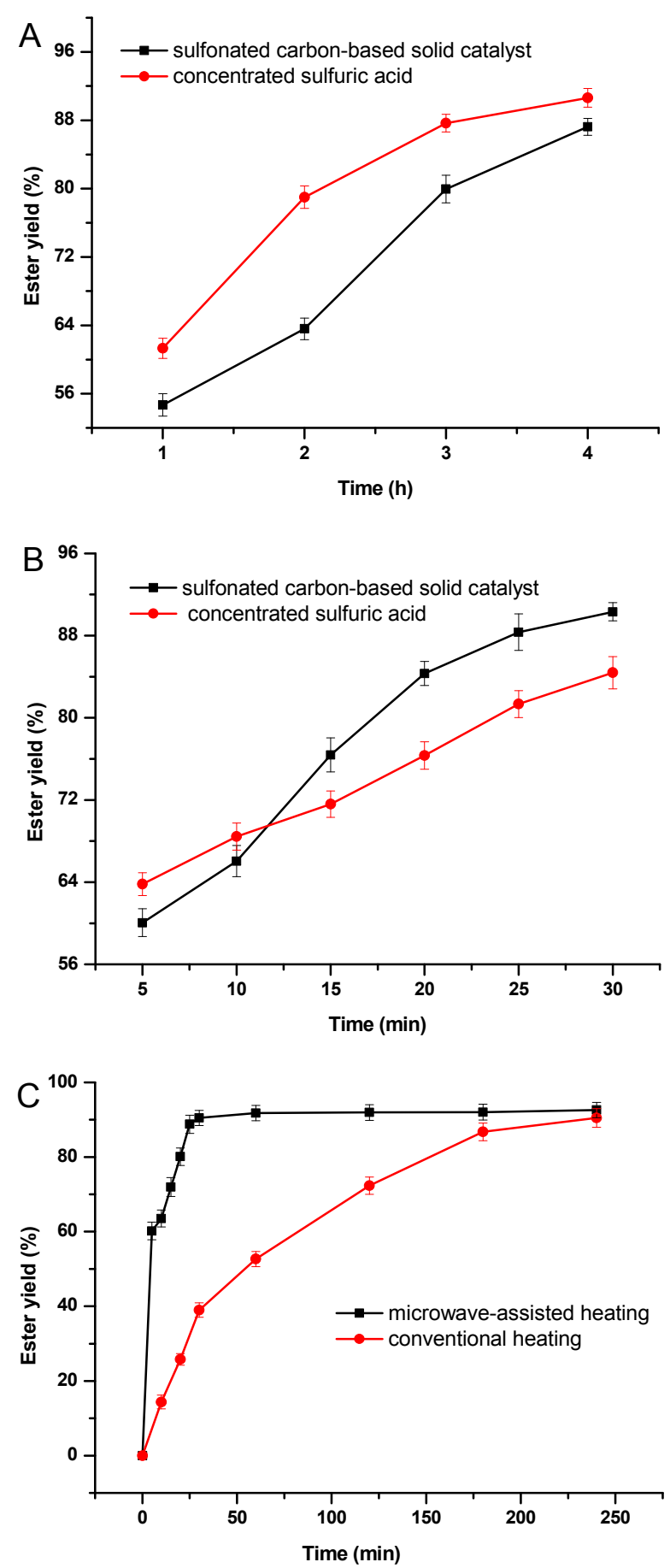

Figure 4. Comparison of ester yield between concentrated sulfuric acid and sulfonated carbon-based solid catalyst. (A) Conventional heating, water bath $60^{\circ} \mathrm{C}$; (B) microwave radiation, microwave power density $1 \mathrm{~W} / \mathrm{mL}$; (C) comparison between conventional and microwave-assisted heating with the sulfonated carbon-based solid catalyst, microwave power density $1 \mathrm{~W} / \mathrm{mL}$, water bath: $60^{\circ} \mathrm{C}$.

The catalytic activity of the two catalysts under microwave radiation was also investigated. As shown in Figure 4B, the sulfonated solid acid catalyst showed higher catalytic activity than the concentrated sulfuric acid, except in the initial reaction period of 0-12 $\mathrm{min}$. This higher catalytic activity was opposite to that under conventional heating environment. This might be attributed to the special irregular porous structure of the sulfonated solid acid catalyst and its polar functional groups such as $-\mathrm{SO}_{3} \mathrm{H}$ and $-\mathrm{COOH}$, which could absorb microwave power and form local hot spots in the 
inside of the catalyst $[16,17]$. Compared to the conventional water-bath heating, the reaction was also completed in a significantly short period (less than $30 \mathrm{~min}$ ), with an ester yield higher than $90 \%$ under microwave heating. All these results illustrated that the current solid catalyst was suitable for the esterification reaction under microwave heating.

As shown in Figure 4C, the reaction reached equilibrium after 30 min using the current solid catalyst with microwave radiation, producing an ester yield of $91.52 \%$. However, the reaction did not yet reach equilibrium after $200 \mathrm{~min}$ with conventional heating. This demonstrated that a higher conversion yield could be obtained in a shorter time with microwave radiation compared to conventional heating (Figure 4C). This phenomenon could also be explained by the following two aspects. Methanol is a polar molecule, and its dipole quickly vibrates and reorientates under microwave irradiation, which could destroy the two-tier structure of the interface between methanol and oleic acid [14]. In addition, the sulfonic acid group $\left(-\mathrm{SO}_{3} \mathrm{H}\right)$ could also be excited by the microwave radiation, causing the local temperature around the $-\mathrm{SO}_{3} \mathrm{H}$ group to be higher than its environment, far exceeding the activation energy needed for esterification. Moreover, this significant decrease in the reaction time could lead to lower production costs. As can be seen in Figure $4 B, C$, the final conversion yields became closer for above two heating methods as the time increased. This indicated that microwave radiation could raise the rate of the esterification reaction while having little effect on the final ester yield.

\subsubsection{Effect of Reusability and Regeneration}

To investigate the reusability and regeneration performance of the current solid catalyst, the used catalyst was collected and washed with hot water and acetone. The recycled catalyst was then subjected to the oleic acid-methanol reaction again. With respect to Table 3, it was not difficult to find that the activity of the catalyst decreased gradually, while the ester yield was maintained at above $60 \%$ after five reaction cycles. This was much higher than that of catalyst made from peanut shell $(50.3 \%)$, while lower than that of catalyst made from rice husk $(75 \%)$, as reported by Zeng et al. $[15,23]$. At the same time, the content of sulfur of the spent solid acid decreased from $7.449 \%$ to $5.152 \%$ and the total acid density decreased from 6.35 to $4.39 \mathrm{mmol} / \mathrm{g}$ (Table 4). These results demonstrated that the $-\mathrm{SO}_{3} \mathrm{H}$ groups in fresh catalysts leached out slowly during the reaction and the leaching out rate would be related to the starting raw materials of catalysts. It has been pointed out elsewhere that the leaching out of $-\mathrm{SO}_{3} \mathrm{H}$ groups was a common problem for sulfonated catalysts [25,26]. More efforts should be made to enhance the stability of $-\mathrm{SO}_{3} \mathrm{H}$ groups in future.

Table 3. Recycling and regeneration performance of the sulfonated carbon-based solid catalyst.

\begin{tabular}{ccc}
\hline \multirow{2}{*}{ Runs } & \multicolumn{3}{c}{ Ester Yield (\%) } \\
\cline { 2 - 3 } & Sulfonated Carbon-Based Solid Catalyst & Regenerated Carbon-Based Solid Catalyst \\
\hline 1 & 91.12 & 87.56 \\
2 & 87.32 & 81.35 \\
3 & 81.05 & 76.07 \\
4 & 75.64 & 68.38 \\
5 & 64.38 & 56.25 \\
\hline
\end{tabular}

Table 4. Textural properties, $\mathrm{S}$ content and total acid density of the samples ${ }^{1}$.

\begin{tabular}{cccccc}
\hline Sample & $S_{\text {BET }}\left(\mathbf{m}^{2} / \mathbf{g}\right)$ & $V_{\text {tot }}\left(\mathbf{c m}^{\mathbf{3}} \mathbf{g}\right)$ & $\boldsymbol{D}(\mathbf{n m})$ & S Content (\%) & Total Acid Density (mmol/g) \\
\hline Solid acid (fresh) & 78.35 & 0.634 & 42.86 & 7.449 & 6.35 \\
Solid acid (spent) & 43.45 & 0.598 & 45.36 & 5.152 & 4.39 \\
Solid acid (regenerated) & 67.32 & 0.602 & 40.28 & 7.158 & 6.10 \\
\hline
\end{tabular}

${ }^{1} S_{\mathrm{BET}}$, specific surface area from Brunauer-Emmett-Teller (BET) method; $V_{\text {tot }}$, total pore volume; $D$, average pore diameter. $\mathrm{S}$ content, elemental $(\mathrm{S})$ content in the sample, measured by elemental analysis. 
The regeneration performance of the current catalyst was also investigated by re-sulfonating it in concentrated sulfuric acid for $1 \mathrm{~h}$ at $110^{\circ} \mathrm{C}$ after being subjected to reaction five times. It was interesting that the activity of the catalyst recovered rapidly through simple re-sulfonation. This result indicated that the current catalyst could be easily regenerated, although the catalytic activity after regeneration was slightly reduced $(87.56 \%)$ relative to the un-recycled performance. At the same time, the total acid density of the regenerated solid acid increased to $6.10 \mathrm{mmol} / \mathrm{g}$, indicating that most of the acid sites could have been recovered by the regeneration process (Table 4).

\subsection{Catalyst Physicochemical Properties Characterization}

XRD patterns for the carbonization carrier and the sulfonated carbon-based solid catalyst are shown in Figure 5A. The two XRD diffraction patterns displayed broad diffraction peaks at $2 \theta=22.086^{\circ}$ and $2 \theta=23.463^{\circ}$ respectively. The broad C (002) diffraction peak indicated that the peak could be attributed to amorphous carbon structures, which was important for the catalytic activity $[19,27]$. There was no noticeable difference in the XRD patterns between the carbonization carrier and sulfonated carbon-based solid catalyst, except for in the latter angle of the diffraction peak, which was shifted to the right by a small amount. This indicated that the process of introducing the sulfonic acid group $\left(-\mathrm{SO}_{3} \mathrm{H}\right)$ to the carbon carrier promoted the further transformation of the carbon structure.
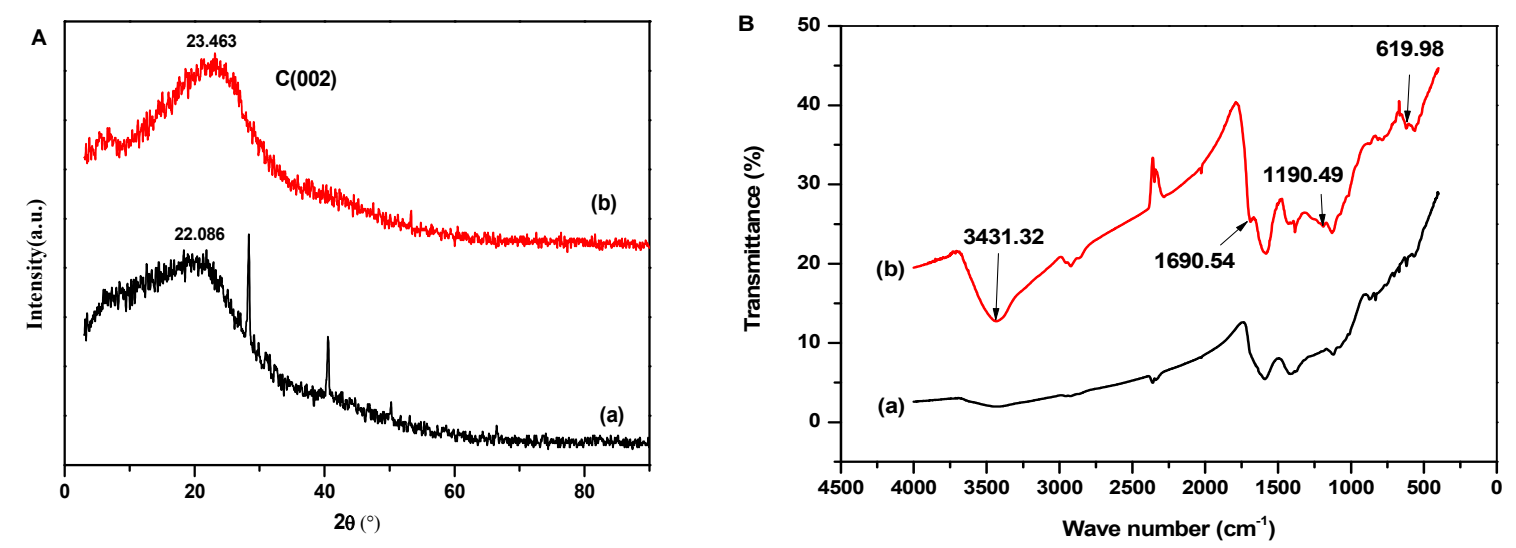

Figure 5. X-ray diffraction (XRD) patterns (A) and Fourier-transform infrared spectra (FT-IR) spectra (B) for carbonized carrier (a) and sulfonated carbon-based solid catalyst (b) prepared from sweet potato.

Figure 5B depicted the FT-IR spectra of the sweet potato catalyst before and after sulfonation. After sulfonation, new absorption peaks emerged for the catalyst at 619.98 and $1190.49 \mathrm{~cm}^{-1}$, corresponding to the characteristic peaks of the sulfonic acid group $\left(-\mathrm{SO}_{3} \mathrm{H}\right)$ and its symmetric stretching vibration of the double bond and asymmetric stretching vibration $[8,28]$. The emergence of the two peaks provided an accurate indication of the presence of sulfonic acid groups in the carbon-based catalyst. Additionally, the bands at 1690.54 and $3431.32 \mathrm{~cm}^{-1}$ were assigned to the $\mathrm{C}=\mathrm{O}$ stretching modes and the $-\mathrm{OH}$ stretching modes of the carboxyl groups, confirming the presence of $-\mathrm{COOH}$ functional groups [6]. Both hydroxyl and carboxyl groups were polar groups closely linked to the strong microwave absorption capacity of the catalyst, which was consistent with the conclusion of the excellent microwave-sensitive property above (Figure 2).

The thermal property of the current catalyst was determined by TGA analysis. The solid catalysts derived from sweet potato showed good thermal stability before and after sulfonation (Figure 6). The small decline $\left(8.78 \%\right.$ ) of the TGA analysis curve in $100-200{ }^{\circ} \mathrm{C}$ for the sweet potato indicated that small gas and moisture molecules escaped, and this process did not change the chemical structure of the sample (Figure 6a). With the increase of temperature $\left(250-400{ }^{\circ} \mathrm{C}\right)$, the weight of the sweet potato declined significantly. The weight loss reached to $56.61 \%$, and the sweet potato entered a thermal decomposition reaction. The chemical bonds of some functional groups, such as ether bonds, hydroxyl 
groups and carboxyl groups, were also broken down. This process was accompanied by a thermal polycondensation reaction, cross-linking cyclization, aromatization and other reactions, which together lead to the formation of a relatively stable polycyclic aromatic structure [29]. The formation of this polycyclic aromatic structure played a crucial role in the subsequent sulfonation process. In addition, the thermogravimetric analysis results for the sweet potato were in accordance with the experimental results for the carbonization temperature (this result was given directly in Section 3.2), indicating that the temperature of the carbonization process should be in the $350-375{ }^{\circ} \mathrm{C}$ range.

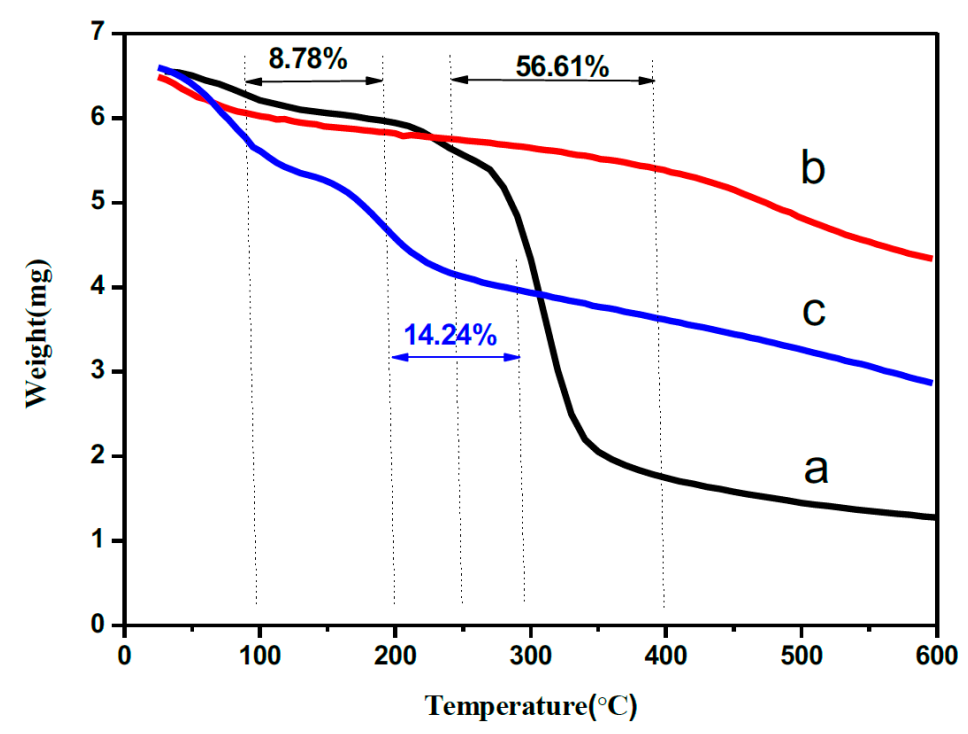

Figure 6. Thermogravimetric analysis (TGA) profiles of sweet potato (a); carbonized carrier (b) and sulfonated carbon-based solid catalyst (c).

Compared to that of the carbonization carrier, the TGA curve of the sulfonated carbon-based solid catalyst showed an extra weightless stage in the $200-300{ }^{\circ} \mathrm{C}$ range (Figure $6 \mathrm{c}$ ). The weight loss was about $14.24 \%$. This phenomenon indicated that the sulfonic acid group $\left(-\mathrm{SO}_{3} \mathrm{H}\right)$ started to decompose and disconnect from the sulfonated samples in this temperature range, and thus would eventually cause the decline of its catalytic activity [8]. Therefore, the esterification reaction temperature should not exceed $200^{\circ} \mathrm{C}$, so as to prevent the deactivation of the catalyst.

The scanning electron microscopy (SEM) images of the sweet potato, carbonized carrier and the carbon-based solid catalyst are displayed in Figure 7. The microstructure changed significantly after incomplete carbonization and sulfonation. As was evident from the SEM image, the raw sweet potato presented a regular spherical and ellipsoidal shape, with little irregular particles around them (Figure 7a). Lots of porous structures and irregular structures appeared after the incomplete carbonization process at high temperature which caused the overflow of water and the chemical reaction of carbohydrates (Figure $7 \mathrm{~b}$ ). After the carbon-based solid acid catalyst formed, the microstructure became looser and more porous, which could be considered as evidence that the catalyst had a large surface area contributing to its catalytic activity (Figure 7c). 

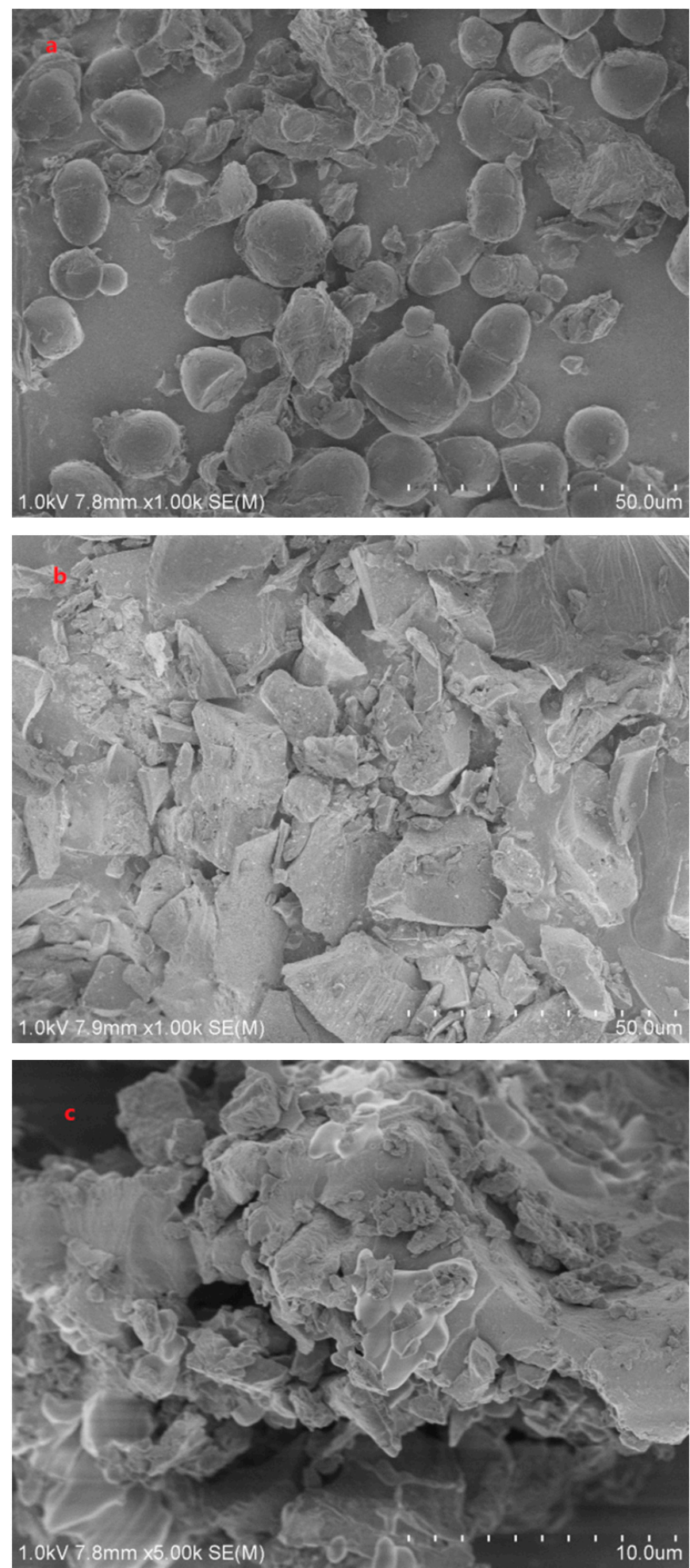

Figure 7. Scanning electron microscope (SEM) images of sweet potato (a); carbonized carrier (b) and sulfonated carbon-based solid catalyst (c).

The EA results of the solid catalyst are summarized in Table 5. The sulfur content was $7.449 \%$ (by mass), which was much higher than that of the sugar catalyst reported by Zong et al. [8]. Combining the TGA, EA FT-IR and SEM analysis results, we can conclude that the sulfonic acid $\left(-\mathrm{SO}_{3} \mathrm{H}\right)$ functional 
group was successfully linked to the carbon carrier after sulfonation and the prepared current solid catalyst had good thermal stability and higher catalytic activity for the relevant reactions $[4,30]$.

Table 5. Elemental analysis of sweet potato, carbonized carrier and sulfonated carbon-based solid catalyst.

\begin{tabular}{ccccc}
\hline Samples & $\mathbf{C} \%$ & $\mathbf{H} \%$ & $\mathbf{O} \%$ & $\mathbf{S} \%$ \\
\hline Sweet potato & 40.46 & 6.484 & 52.329 & 0 \\
Carbonization carrier & 69.80 & 4.052 & 24.859 & 0 \\
Sulfonated carbon-based catalyst & 47.88 & 4.471 & 39.376 & 7.449 \\
\hline
\end{tabular}

\section{Materials and Methods}

\subsection{Materials}

Methanol, oleic acid, sulfuric acid, ether, ethanol, phenolphthalein and potassium hydroxide were purchased from Sino-Pharm Chemical Reagent Co., Ltd. (Shanghai, China). All reagents were analytic grade. The sweet potato was purchased from a local market (Wuxi, China). The amylose content of the sweet potato was determined to be $16.6 \%$ of total starch.

\subsection{Catalyst Preparation}

The catalyst was prepared according to the method described by Zeng et al. [15], with partial improvement. The fresh sweet potatoes were washed and peeled, then sliced into 3-5 $\mathrm{mm}$ pieces. The sliced sweet potato flesh was dried in an oven at $70^{\circ} \mathrm{C}$ for $1 \mathrm{~h}$. After dehydration, the sweet potato was carbonized in a muffle furnace at high temperatures $\left(300-400{ }^{\circ} \mathrm{C}\right)$ for a set time $(20-60 \mathrm{~min})$ to obtain the incompletely carbonized solids. These solids were then ground into powder and sulfonated using concentrated sulfuric acid ( $>98 \%)$ at the ratio of $1 \mathrm{~g} / 10 \mathrm{~mL}$ at $70-130{ }^{\circ} \mathrm{C}$ for $1-5 \mathrm{~h}$. After cooling to room temperature, the black precipitate was collected by vacuum filtration and rinsed thoroughly with hot distilled water $\left(100^{\circ} \mathrm{C}\right)$ to remove impurities such as sulfate ions. The black solid catalyst was then dried in an oven $\left(70^{\circ} \mathrm{C}\right)$ with a thermostat blast.

The factors affecting catalytic activity included the carbonization and sulfonation temperatures and times, which were thoroughly investigated and optimized. The final catalyst preparation condition was carbonization at $375^{\circ} \mathrm{C}$ for $40 \mathrm{~min}$ and sulfonation at $110^{\circ} \mathrm{C}$ for $1 \mathrm{~h}$.

\subsection{Investigation of Microwave-Sensitive and Homogeneous Properties}

The homogenous property of microwave heating was examined by using the model of $20 \mathrm{~g}$ of oleic acid with $1 \mathrm{~g}$ of catalyst under microwave power of $100 \mathrm{~W}$ for $10 \mathrm{~min}$. The tested mixture was placed in the nine different points as shown in Figure 1A. Then the temperature was recorded and drawn to reflect the homogeneous property of microwave heating.

The microwave-sensitive property was investigated by using the model of $20 \mathrm{~g}$ of oleic acid with/without $1 \mathrm{~g}$ catalyst under different microwave power (100, 200, and $300 \mathrm{~W}$ ) for $10 \mathrm{~min}$. The temperature of the model under microwave heating was recorded at the interval of $1 \mathrm{~min}$. The heating rate $(k)$ was calculated and reflected as the microwave-sensitive property of the catalyst.

\subsection{Catalytic Esterification Activity Measurements}

The catalytic esterification activity was measured using the model of methanol and oleic acid with a molar ratio of $6: 1$ and a catalyst concentration of $5 \%$. Methanol $(99.5 \mathrm{wt} \%)$, oleic acid and the catalyst were fed into a $250-\mathrm{mL}$ three-necked round-bottomed quartz flask, and a reflux condenser was used to condense the methanol. The conventional heating reactions were carried out using a digital heating circulating water bath with mechanical stirring $(200 \mathrm{rpm})$. The microwave radiation reactor (Nanjing Maigewei Process Equipment Technology Co., Ltd., Nanjing, China), operated at $2450 \mathrm{MHz}$, 
was power programmable from 0 to $850 \mathrm{~W}$. Mechanical stirring was used at a rotate speed adjustable from 0 to $600 \mathrm{rpm}$ to homogenize the suspension. A fiber optic sensor was hung into reactor and used to check the reaction temperature across time. The mode of microwave irradiation was non-pulsed continuous microwave heating. The microwave heating power was controlled by automatic feedback with the temperature or using fixed values, and the fluctuation range of the reaction temperature was $6{ }^{\circ} \mathrm{C}$.

The acid values of the products were determined according to BS EN ISO 660 (National Standard of the People's Republic of China. GB/T5530-1998). The ester yield of the reaction was determined based on the changes in the acid value in the oil phase and calculated as follows:

$$
\text { The ester yield }(\%)=\frac{A V_{o}-A V_{p}}{A V_{o}} \times 100 \%
$$

where, $A V_{o}$ and $A V_{p}$ were the acid values of the oleic acid and the products, respectively.

The catalysts recycled from the reaction system were washed with acetone for repetitive usability analysis. Regeneration analysis was also performed by re-sulfonating the catalysts with concentrated sulfuric acid. The catalytic activity was tested with both conventional and microwave heating.

\subsection{Physicochemical Properties Characterization}

The X-ray diffraction (XRD) analysis was performed in an X-ray diffractometer (Model: D8, Bruker AXS Ltd., Karlsruhe, Germany) equipped with $\mathrm{Cu}-\mathrm{K} \alpha(\lambda=1.54178 \AA)$ radiation and a scanning angle $(2 \theta)$ of $2^{\circ}-90^{\circ}$ at a scanning speed of $4^{\circ} / \mathrm{min}$. The Brunauer-Emmett-Teller (BET) specific surface areas and the total pore volumes of the samples were measured by a Micromeritics ASAP2020 analyzer (MICROMERITICS INSTRUMENT CORP, Atlanta, GA, USA) with nitrogen adsorption-desorption at $-196{ }^{\circ} \mathrm{C}$. The total acid site density of the samples was obtained by titration method in aqueous solution. The qualitative analysis of the catalyst was carried out using a Fourier transform infrared spectrometer (Model: Nicolet iS10, Thermo Fisher Corporation, Madison, WI, USA). The thermal stability of the catalyst was characterized using a thermogravimetric analyzer (Model: TGA/SDTA851e, Mettler Toledo Instruments Co., Ltd., Zurich, Switzerland) under flowing nitrogen with a heating rate of $10^{\circ} \mathrm{C} / \mathrm{min}$ from 25 to $800{ }^{\circ} \mathrm{C}$. The composition of the catalyst elements was determined using Elementar (Model: VARIOEL III, Elementar Vario, Langenselbold, Germany). The morphology of the prepared catalysts was determined using a scanning electron microscope (SEM, Model: S-4800, Hitachi Co., Ltd., Tokyo, Japan) with an accelerating voltage of $15 \mathrm{kV}$.

\section{Conclusions}

In summary, the catalyst could be simply prepared from sweet potato and was confirmed to have favorable microwave-sensitive and homogeneous properties, with at least two Bronstein acid sites: $-\mathrm{COOH}$ and $-\mathrm{SO}_{3} \mathrm{H}$ groups. The loose and porous microstructure and high content of elemental sulfur $(7.449 \%)$ ensured the high catalytic activity $(>91 \%)$ in the oleic acid-methanol esterification reaction within $30 \mathrm{~min}$ with microwave power density of $1.0 \mathrm{~W} / \mathrm{mL}$. However, the reaction temperature should not exceed $200{ }^{\circ} \mathrm{C}$. The catalyst deactivated gradually after multiple cycles of use, but could be regeneration by simple re-sulfonation.

Acknowledgments: This work was financially supported by National Natural Science Foundation of China (No. 31401493), the Fundamental Research Funds for the Central Universities (Nos. JUSRP51511-1 and JUSRP51634B).

Author Contributions: Zheng-Wei Cui and Hai-Ying Chen conceived and designed the experiments; Zheng-Wei Cui contributed reagents/materials/analysis tools; Hai-Ying Chen performed the experiments, analyzed the data and wrote the paper.

Conflicts of Interest: The authors declare no competing financial interest. 


\section{References}

1. Chai, F.; Cao, F.H.; Zhai, F.Y.; Chen, Y.; Wang, X.H.; Su, Z.M. Transesterification of vegetable oil to biodiesel using a heteropolyacid solid catalyst. Adv. Synth. Catal. 2007, 349, 1057-1065. [CrossRef]

2. Kiss, A.A.; Dimian, A.C.; Rothenberg, G. Solid acid catalysts for biodiesel production-towards sustainable energy. Adv. Synth. Catal. 2006, 348, 75-81. [CrossRef]

3. Lotero, E.; Liu, Y.J.; Lopez, D.E.; Suwannakarn, K.; Bruce, D.A.; Goodwin, J.G. Synthesis of biodiesel via acid catalysis. Ind. Eng. Chem. Res. 2005, 44, 5353-5363. [CrossRef]

4. Takagaki, A.; Toda, M.; Okamura, M.; Kondo, J.N.; Hayashi, S.; Domen, K.; Hara, M. Esterrification of higher fatty acids by a novel strong solid acid. Catal. Today 2006, 116, 157-161. [CrossRef]

5. Vicente, G.; Martinez, M.; Aracil, J. Integrated biodiesel production: A comparison of different homogeneous catalysts systems. Bioresour. Technol. 2004, 92, 297-305. [CrossRef] [PubMed]

6. Chen, G.; Fang, B. Preparation of solid acid catalyst from glucose-starch mixture for biodiesel production. Bioresour. Technol. 2011, 102, 2635-2640. [CrossRef] [PubMed]

7. Toda, M.; Takagak, A.; Okatnura, M.; Kondo, J.N.; Hayashi, S.; Domen, K.; Hara, M. Green chemistry-Biodiesel made with sugar catalyst. Nature 2005, 438, 178. [CrossRef] [PubMed]

8. Zong, M.H.; Duan, Z.Q.; Lou, W.Y.; Thomas, J.S.; Wu, H. Preparation of a sugar catalyst and its use for highly efficient production of biodiesel. Green Chem. 2007, 9, 434-437. [CrossRef]

9. Su, F.; Guo, Y.H. Advancements in solid acid catalysts for biodiesel production. Green Chem. 2014, 16, 2934-2957. [CrossRef]

10. Kappe, C.O. Controlled microwave heating in modern organic synthesis. Angew. Chem. Int. Ed. 2004, 43, 6250-6284. [CrossRef] [PubMed]

11. Hemando, J.; Leton, P.; Matia, M.P.; Novella, J.L.; Alvarez-Builla, J. Biodiesel and FAME synthesis assisted by microwaves: Homogeneous batch and flow processes. Fuel 2007, 86, 1641-1644.

12. Leadbeater, N.E.; Stencel, L.M. Fast, easy, preparation of biodiesel using microwave heating. Energy Fuel 2006, 20, 2281-2283. [CrossRef]

13. Motasemi, F.; Ani, F.N. A review on microwave-assisted production of biodiesel. Renew. Sustain. Energy Rev. 2012, 16, 4719-4733. [CrossRef]

14. Yuan, H.; Yang, B.L.; Zhu, G.L. Synthesis of biodiesel using microwave absorption catalysts. Energy Fuel 2009, 23, 548-552. [CrossRef]

15. Zeng, D.; Liu, S.; Gong, W.; Wang, G.; Qiu, J.; Chen, H. Synthesis, characterization and acid catalysis of solid acid from peanut shell. Appl. Catal. A 2014, 469, 284-289. [CrossRef]

16. Wang, X.; Liu, R.; Waje, M.M.; Chen, Z.; Yan, Y.; Bozhilov, K.N.; Feng, P. Sulfonated ordered mesoporous carbon as a stable and highly active protonic acid catalyst. Chem. Mater. 2007, 19, 2395-2397. [CrossRef]

17. Zhang, S.; Zu, Y.G.; Fu, Y.J.; Luo, M.; Zhang, D.Y.; Efferth, T. Rapid microwave-assisted transesterification of yellow horn oil to biodiesel using a heterpolyacid solid catalyst. Bioresour. Technol. 2011, 101, 931-936. [CrossRef] [PubMed]

18. Lou, W.Y.; Zong, M.H.; Duan, Z.Q. Efficient production of biodiesel from high free fatty acid-containing waste oils using various carbohydrate-derived solid acid catalysts. Bioresour. Technol. 2008, 99, 8752-8758. [CrossRef] [PubMed]

19. Liu, X.Y.; Huang, M.; Ma, H.L.; Zhang, Z.Q.; Gao, J.M.; Zhu, Y.L.; Han, X.J.; Guo, X.Y. Preparation of a carbon-based acid catalyst by sulfonating activated carbon in a chemical reduction process. Molecules 2010, 15, 7188-7196. [CrossRef] [PubMed]

20. Wang, M.W.; Wu, W.W.; Wang, S.S.; Shi, X.Y.; Wu, F.A.; Wang, J. Preparation and characterization of a solid acid catalyst from macro Fungi residue for methyl palmitate production. BioResources 2015, 10, 5691-5708. [CrossRef]

21. Liu, T.T.; Li, Z.L.; Li, W.; Shi, C.J.; Wang, Y. Preparation and characterization of biomass carbon-based solid acid catalyst for the esterification of oleic acid with methanol. Bioresour. Technol. 2013, 133, 618-621. [CrossRef] [PubMed]

22. Wang, L.H.; Liu, H.; Li, L. Carbon-based acid catalyst from waste seed shells: Preparation and characterization. Pol. J. Chem. Technol. 2015, 17, 37-41. [CrossRef]

23. Zeng, D.L.; Zhang, Q.; Chen, S.Y.; Liu, S.L.; Wang, G.H. Synthesis porous carbon-based solid acid from rice husk for esterification of fatty acids. Microporous Mesoporous Mater. 2016, 219, 54-58. [CrossRef] 
24. Geng, L.; Wang, Y.; Yu, G.; Zhu, Y.X. Efficient carbon-based solid acid catalysts for the esterification of oleic acid. Catal. Commun. 2011, 13, 26-30. [CrossRef]

25. Mo, X.; Lopez, D.E.; Suwannakarn, K.; Liu, Y.; Lotero, E.; Goodwin, J.G.; Lu, C.Q. Activation and deactivation characteristics of sulfonated carbon catalysts. J. Catal. 2008, 254, 332-338. [CrossRef]

26. Suppalakpanya, K.; Ratanawilai, S.B.; Tongurai, C. Production of ethylester from esterified crude palm oil by microwave with dry washing by bleaching earth. Appl. Energy 2010, 87, 2356-2359. [CrossRef]

27. Tsubouchi, N.; Xu, C.B.; Ohtsuka, Y. Carbon crystallization during high-temperature pyrolysis of coals and the enhancement by calcium. Energy Fuel 2003, 17, 1119-1125. [CrossRef]

28. Liang, X.Z.; Yang, J.G. Sythesis of a novel carbon based strong acid catalyst through hydrothermal carbonization. Catal. Lett. 2009, 132, 460-463. [CrossRef]

29. Soares, S.; Camino, G. Comparative study of the thermal decomposition of pure cellulose and pulp paper. Polym. Degrad. Stab. 1995, 49, 275-283. [CrossRef]

30. Devi, B.A.P.; Gangadhar, K.N.; Prasad, P.S.P.; Jagannadh, B.; Prasad, R.B.N. A glycerol-based carbon catalyst for the preparation of biodiesel. ChemSusChem 2009, 2, 617-620. [CrossRef] [PubMed]

(C) 2016 by the authors; licensee MDPI, Basel, Switzerland. This article is an open access article distributed under the terms and conditions of the Creative Commons Attribution (CC-BY) license (http://creativecommons.org/licenses/by/4.0/). 